\title{
Ermittlung spezifischer Materialkennwerte von Schichten mittels Interdigital-Elektroden
}

\author{
Jörg Exner und Ralf Moos \\ Lehrstuhl für Funktionsmaterialien, Universität Bayreuth, Deutschland \\ Kontakt: funktionsmaterialien@uni-bayreuth.de
}

\section{Zusammenfassung}

Eine einfache aber genaue Ermittlung von spezifischen Materialkennwerten ist nicht nur für die Materialforschung, sondern auch in der späteren Anwendung von großem Interesse. Gerade Interdigital-Elektroden ermöglichen ein besonders einfaches Aufbringen der Beschichtung, da keine Deckelelektrode benötigt wird, erschweren aber im Gegenzug die Bestimmung von elektrischen Materialkennwerten durch einen komplexen, zunächst unbekannten Verlauf der Feldlinien. In dieser Arbeit wird deshalb ein einfach anzuwendender und nur von der Dicke der Schicht abhängiger Korrekturfaktor $F_{\text {geo }}$ vorgestellt, welcher durch eine Modellierung und Simulation mit der Software COMSOL Multiphysics ermittelt wurde. Eine Validierung erfolgt anhand von siebgedruckten bzw. per Aerosoldeposition (ADM) erzeugten ionenleitfähigen 8YSZ-Schichten verschiedener Dicken. Die ermittelten Leitfähigkeiten $\sigma$ stimmen für alle Schichtdicken gut mit der Literatur überein.

Keywords: Interdigital-Elektroden, spezifische Materialkennwerte, Simulation, Korrekturfaktor

\section{Einleitung}

Die genaue Bestimmung elektrischer Kennwerte von Schichten (z.B. die Leitfähigkeit $\sigma$ oder die Permittivität $\varepsilon_{r}$ ) ist für viele Anwendungen in der Sensorik [1] und Materialforschung [2] von großem Interesse. Um zu vermeiden, dass auf die Schichten eine Deckelektrode aufgebracht werden muss, können alternativ Schichten auf InterdigitalElektroden (IDE) aufgebracht werden. Hierfür wird das zu beschichtende Substrat zuerst mit der Interdigital-Elektrode z.B. mittels Siebdruck oder einem strukturierten Dünnschichtprozess versehen, und anschließend der gegebenenfalls notwendige Einbrenn- bzw. Auslagerungsschritt durchgeführt. Die abschließend aufgebrachte Beschichtung kann danach im Idealfall direkt elektrisch vermessen werden, ohne weitere Prozessschritte erforderlich zu machen. Folglich können auch temperaturempfindliche, mechanisch anfällige oder poröse Beschichtungen untersucht werden.

Will man damit nicht nur geometrieabhängige Impedanzen $\underline{Z}$, sondern auch spezifische Materialkenngrößen bestimmen, muss eine genaue Feldverteilung bekannt sein. Zwar ist dieses Thema bereits oft in der Literatur behandelt worden, jedoch soll hier ein relativ einfach anzuwendendes Verfahren vorgestellt werden. Dieses verwendet lediglich einen simulationsbasierten Korrekturfaktor bzw. Geometriefaktor $F_{\text {geo }}$, der nur von der Elekt- rodengeometrie und der Schichtdicke abhängig ist. Damit ist dann ein einfaches Bestimmen elektrischer Materialkennwerte möglich.

\section{Simulation}

Für die Modellierung der elektrischen Feldlinien an der Interdigital-Elektrode inklusive Beschichtung und Substrat wurde das AC/DC-Modul der Software COMSOL Multiphysics verwendet.

Berechnungen erfolgten exemplarisch für eine Elektrodenstruktur, bestehend aus den Zuleitungen für Elektrode $A$ und $B$ (Abb. 1) und jeweils 15 Fingern mit einer Länge von 4,7 mm, einer Breite von $100 \mu \mathrm{m}$ und einem Abstand zwischen den Fingern von ebenfalls $100 \mu \mathrm{m}$ (siehe Detailzeichnung).

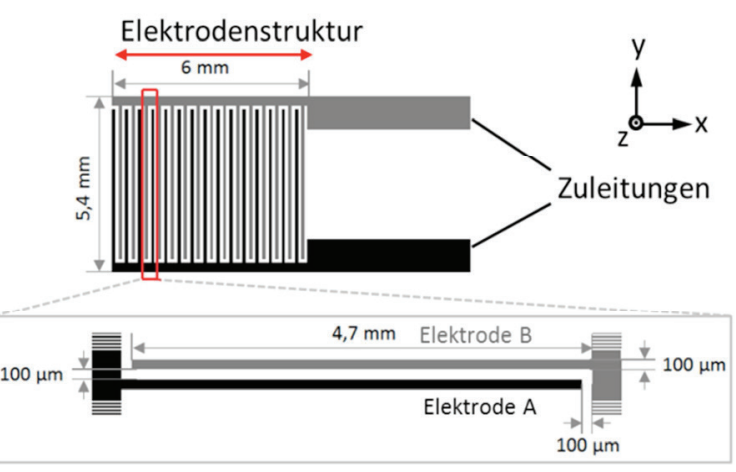

Abb. 1: Geometrie der Interdigital-Elektrode (Aufsicht) 
Unter dieser Struktur befindet sich ein Aluminiumoxid-Substrat mit einer Dicke von $630 \mu \mathrm{m}$, oberhalb die Beschichtung mit variierender Schichtdicke (1 $\mu \mathrm{m}-2 \mathrm{~mm})$. Abschließend enthält das Modell eine $4 \mathrm{~mm}$ hohe Luftschicht oberhalb der Probe. Ein Querschnitt des Modells ist in Abb. 2 dargestellt.

Der Korrekturfaktor $F_{\text {geo }}$ wurde parallel über zwei verschiedene Untermodule ermittelt, um die Verlässlichkeit der Ergebnisse zu erhöhen. Hierzu wurde einerseits die gesamte in Abb. 1 dargestellte Struktur mit einer darauf befindlichen leitfähigen Beschichtung (mit variierenden vorgegebenen Leitfähigkeiten $\left.\sigma_{\text {Schicht,Modell }}\right)$ unter Verwendung des Untermoduls electric current (EC) simuliert. Dazu wurde als Randbedingung an den Zuleitungen eine Potentialdifferenz $U_{S}$ vorgegeben und der resultierende Strom $I_{C}$ berechnet und daraus der simulierte Widerstand $R_{\mathrm{c}}$ ermittelt.

Andererseits wurde nur ein Elektrodenpaar, dargestellt wie in der Detailzeichnung von Abb. 1, mit Hilfe des electro static (ES) Untermoduls simuliert, wobei die modellierte Schichtdicke und die vorgegebene Permittivität $\varepsilon_{r, \text { Modell }}$ variiert wurden.

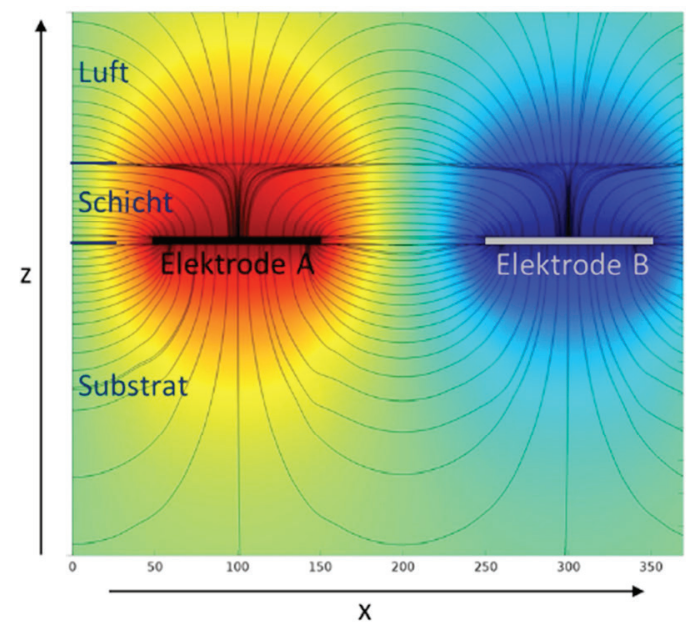

Abb. 2: E-Feld-Simulation eines Elektrodenpaares für ein dielektrisches Beschichtungsmaterial: Potential (farbig) und Verlauf der Feldlinien (schwarz)

Die für dieses Elektrodenpaar ermittelte Einzelkapazität wurde anschließend auf die Gesamtkapazität $C$ der Parallelschaltung umgerechnet. Für die Auswertung wurde die vorgegebene Permittivität $\varepsilon_{\mathrm{r}, \text { Modell }}$ der Schicht mit der berechneten Kapazität korreliert (analoge Vorgehensweise bei $\sigma$ und $G=1 / R)$.

\section{Experimentelle Durchführung}

Die Simulationsergebnisse wurden anhand von ionisch leitfähigen YSZ-Schichten auf Goldelektro- den (Höhe $5 \mu \mathrm{m}$, Abstand und Breite der Elektroden je $100 \mu \mathrm{m}$ ) überprüft. Hierzu wurden 8YSZSchichten verschiedener Dicke per Siebdruck hergestellt und bei $1270{ }^{\circ} \mathrm{C}$ eingebrannt. Zur Einstellung der Schichtdicke wurden die Substrate zwischen einem und 4 Mal bedruckt, was zu Schichtdicken (nach dem Einbrennen) zwischen 7 und $39 \mu \mathrm{m}$ führte (siehe Abb. 3).

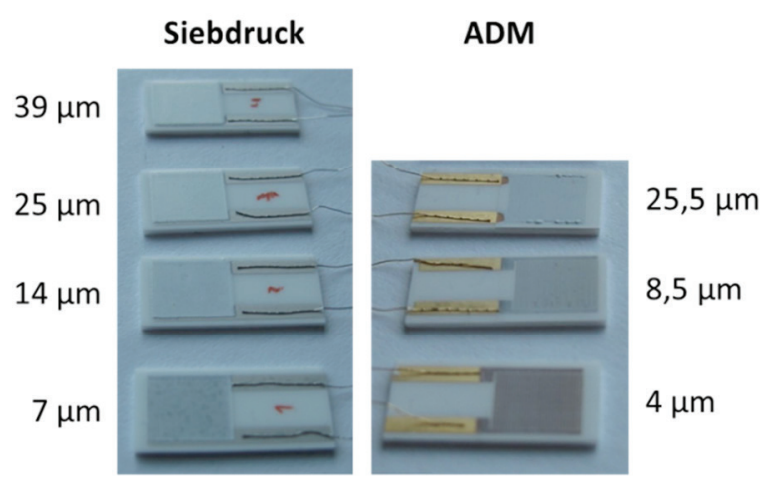

Abb. 3: Übersicht über die per Siebdruck und ADM hergestellten 8YSZ-Schichten

Da siebgedruckte Schichten oft porös sind, wurden zusätzlich drei Proben per Aerosoldeposition (ADM) hergestellt, welche die Abscheidung von dichten keramischen Schichten bei Raumtemperatur ermöglicht. Eine genauere Funktionsweise der ADM und die hierfür eingesetzte Beschichtungsanlage sind in [3][4] beschrieben. Per ADM erzeugte Schichten hatten eine Dicke zwischen 4 und $25 \mu \mathrm{m}$ (Abb. 3). Vor der elektrischen Vermessung wurden die Schichten bei $800{ }^{\circ} \mathrm{C} 5 \mathrm{~h}$ lang getempert.

Alle hergestellten 8YSZ-Schichten wurden in einem mit synthetischer Luft gespülten Aluminiumoxid-Rohrofen bei 400, 500 und $600{ }^{\circ} \mathrm{C}$ per Impedanzspektroskopie (Anregungsspannung $U_{\text {rms }}$ $50 \mathrm{mV}$, Frequenz $1 \mathrm{~Hz}$ bis $10 \mathrm{MHz}$ ) elektrisch vermessen. Der Gesamtwiderstand $R$ wurde aus Nyquist-Plots der gemessenen Impedanz $\underline{Z}$ abgelesen. Anschließend wurde daraus die Leitfähigkeit $\sigma$ mittels gemessener Schichtdicke und dem hier vorgestellten Korrekturfaktor $F_{\text {geo }}$ ermittelt.

\section{Ergebnisse und Diskussion}

Mit Hilfe des simulierten Korrekturfaktors $F_{\text {geo }}$ können aus den gemessenen Werten $R$ und $C$ die Materialkennwerte $\sigma$ und $\varepsilon_{\mathrm{r}}$ über die Gleichungen (1) bzw. (2) ermittelt werden:

$$
\begin{aligned}
& \sigma=\frac{1}{R \cdot F_{\text {geo }}} \\
& \varepsilon_{\mathrm{r}}=\frac{C_{\text {Schicht }}}{F_{\text {geo }} \cdot \varepsilon_{0}}+1
\end{aligned}
$$


Die hierbei benötigte Kapazität der Schicht $C_{\text {Schicht }}$ bestimmt sich aus der Differenz der gemessenen Kapazität $C$ mit und ohne Beschichtung.

Der für leitfähige Schichten mittels $E C$-Untermodul und für dielektrische Schichten mittels $E S$ Untermodul bestimmte Korrekturfaktor $F_{\text {geo }}$ ist für beide simulierten Wege identisch, womit die prinzipielle Gültigkeit der Modellierung gezeigt werden kann. Für den IDE mit der zugrunde liegenden Struktur aus Abb. 1 ergeben sich bei $F_{\text {geo }}$ drei verschiedene Bereiche in Abhängigkeit der Schichtdicke (Abb. 4).

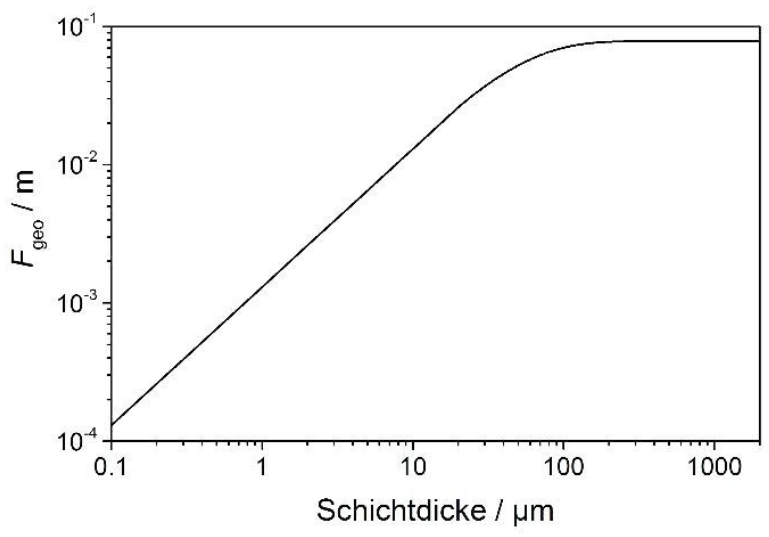

Abb. 4: Korrekturfaktor für den IDE mit $100 \mu \mathrm{m}$ Elektrodenbreite und -abstand

Für dünne Schichten bis $20 \mu \mathrm{m}$ nimmt $F_{\text {geo }}$ in der doppellogarithmischen Darstellung zunächst linear mit steigender Schichtdicke zu. In diesem Bereich stimmt $F_{\text {geo }}$ mit der analytischen Berechnung [5] unter Annahme ausschließlich parallel verlaufender Feldlinien zwischen den Elektroden A und B überein. Eine Verdoppelung der Schichtdicke führt direkt zu einer Verdoppelung des vom Strom durchflossenen Querschnitts und damit zu einer identischen Zunahme von $F_{\text {geo }}$.

Übersteigt die Schichtdicke nun das 0,2 fache des Elektrodenabstandes (hier $20 \mu \mathrm{m}$ ), nimmt die Steigung zu höheren Schichtdicken zunehmend ab und die Abweichung zur analytischen Lösung nimmt zu. Die Feldliniendichte in den oberen Teilen der Beschichtung nimmt ab, da die Feldlinien hier deutlich verlängert sind im Vergleich zum direkten Abstand zwischen den beiden Elektroden.

Für Schichtdicken oberhalb des doppelten Elektrodenabstandes $(200 \mu \mathrm{m})$ ist der Korrekturfaktor $F_{\text {geo }}$ dann annähernd konstant, da die Feldliniendichte hier vernachlässigbar klein wird. In diesem Bereich stimmt $F_{\text {geo }}$ sehr gut mit dem an Wassertropfen bestimmten Geometriefaktor von 0,07 $\mathrm{m}$ überein [6]. Der mittels Simulation bestimmte Korrekturfaktor passt somit sehr gut zu den bisher veröffentlichten Umrechnungsansätzen für kleine und sehr große Schichtdicken, erweitert jedoch den Gültig- keitsbereich auf die interessanten mittlere Schichtdickenbereiche zwischen 20 und $200 \mu \mathrm{m}$.

Für die bei 400,500 und $600{ }^{\circ} \mathrm{C}$ untersuchten 8YSZ-Beschichtungen wurde nun mit Hilfe des Korrekturfaktors aus den gemessenen Widerständen $R$ die Leitfähigkeit $\sigma$ ermittelt. In Abb. 5a ist $\sigma$ der ADM-Schichten in Abhängigkeit der Temperatur $T$ (Arrhenius-artige Darstellung) gezeigt. Zusätzlich sind Bulk-Werte aus [7] eingezeichnet. Alle ADM-Schichten zeigen in Folge des Geometriefaktors unabhängig von der Schichtdicke gleiche Leitfähigkeiten, welche gut mit Bulk-Werten übereinstimmen.

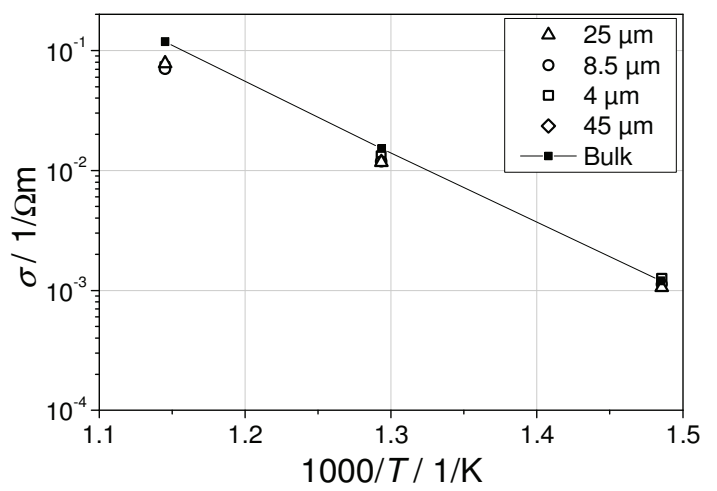

a) mittels $A D M$ hergestellt

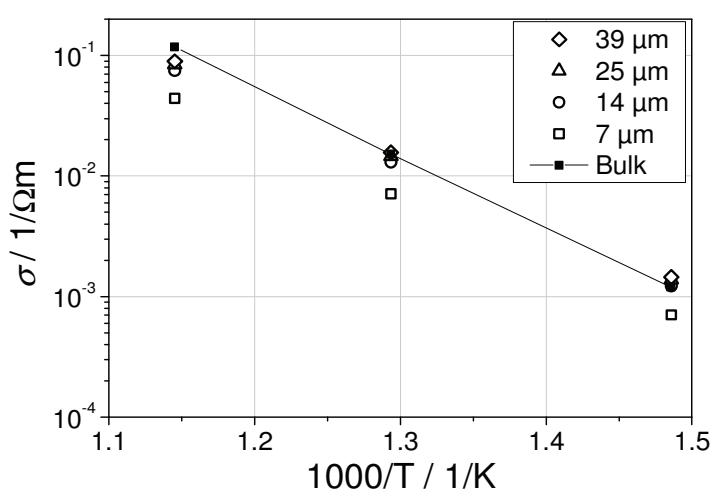

b) siebgedruckte Schichten

Abb. 5: Arrhenius-artige Darstellung der Leitfähigkeit $\sigma$ von 8YSZ-Schichten für verschiedene Schichtdicken

Die siebgedruckten Schichten folgen dem gleichen Verlauf wie ADM-Schichten. Mit Ausnahme der Probe mit $7 \mu \mathrm{m}$ Schichtdicke stimmen die gemessenen Leitfähigkeiten der Proben überein. Die Probe mit $7 \mu \mathrm{m}$ Schichtdicke fällt etwas nach unten hin $a b$, was jedoch mit einer ungleichmäßigen Morphologie begründet werden kann.

Mit Hilfe des Geometriefaktors können folglich Leitfähigkeiten einfach und genau an InterdigitalElektroden gemessen werden. 


\section{Literatur}

[1] GROSS, A.; HANFT, D.; BEULERTZ, G.; MARR, I.; KUBINSKI, D. J.; VISSER, J. H.; MOOS, R.: The effect of $\mathrm{SO}_{2}$ on the sensitive layer of a $\mathrm{NO}_{x}$ dosimeter. In: Sensors and Actuators B: Chemical 187 (2013), S. 153-161, doi: 10.1016/j.snb.2012.10.039

[2] SHIBUYA, M.; NISHINA, T.; MATSUE, T.; UCHIDA, I.: In Situ Conductivity Measurements of $\mathrm{LiCoO}_{2}$ Film during Lithium Insertion/Extraction by Using Interdigitated Microarray Electrodes. In: Journal of The Electrochemical Society 143 (1996), S. 31573160, doi: $10.1149 / 1.1837180$

[3] EXNER, J.; FUIERER, P.; MOOS, R.: Aerosol deposition of $(\mathrm{Cu}, \mathrm{Ti})$ substituted bismuth vanadate films. In: Thin Solid Films 573 (2014), S. 185-190, doi: 10.1016/j.tsf.2014.11.037.

[4] HANFT, D.; EXNER, J.; SCHUBERT, M.; STÖCKER, T.; FUIERER, P.; MOOS, R.: An Overview of the Aerosol Deposition Method: Process Fundamentals and New Trends in Materials Applications. In: J. Cerm. Sci. Technol. 6 (2015), S. 147-182, doi: 10.4416/JCST201500018.

[5] SHU, J. H.; WIKLE, H. C.; CHIN, B. A.: Passive chemiresistor sensor based on iron (II) phthalocyanine thin films for monitoring of nitrogen dioxide. In: Sensors and Actuators B: Chemical 148 (2010), S. 498-503, doi: 10.1016/j.snb.2010.05.017.

[6] SCHÖNAUER, D.; MOOS, R.: Detection of water droplets on exhaust gas sensors. In: Sensors and Actuators B: Chemical 148 (2010), S. 624-629. doi: 10.1016/j.snb.2010.05.060.

[7] GHATEE, M.; SHARIAT, M. H.; IRVINE, J. T. S.: Investigation of electrical and mechanical properties of 3YSZ/8YSZ composite electrolytes. In: Solid State Ionics 180 (2009), S. 5762. doi: 10.1016/j.ssi.2008.10.006. 